\title{
Automatic role-oriented assignment of channels in the ad hoc network of hierarchically organized agents
}

\author{
Alexander Kuznetsov \\ Voronezh State University \\ 394036, Universiteskaja pl., 1 \\ Voronezh, Russia \\ avkuz@bk.ru
}

\begin{abstract}
The work deals with the construction of the automatic channel assignment algorithm in which channels from the given set of channels distributed between agents, organized in hierarchically nested groups. The channels are assigned in respect the agent role and position in the hierarchy. We formulated the special resources allocation problem with repelling and attracting of the agents to describe the channel assignment. Also we demonstrated the application of the algorithm on an example of a self-organizing ad hoc mobile network. Estimations on the time of the organization of the network of agents are given.
\end{abstract}

\section{CCS Concepts}

-Networks $\rightarrow$ Mobile ad hoc networks; •Applied computing $\rightarrow$ Cyberwarfare; $\bullet$ Computing methodologies $\rightarrow$ Multi-agent systems;

\section{Keywords}

software-defined network, self-organizing agent, hierarchical network, automatic distribution of channels

\section{INTRODUCTION}

In this paper, a resource allocation problem is considered whereby system include agents, resources and signals. Each agent can search and capture resource. Agents are assumed to be different kinds and they can aggregate into groups of different sizes. Moreover, each agent can capture a resource. Also every resource have the maximum limit of agents which can use this resource simultaneously. The agent also can transmit a signal after resource capturing to other agents. This signals include some agent's and resource's characteristics and can attract to the resource other agents of the same kind as the given agent and repel agents of the another kind. Our goal is to force agents to transmit such signals that the resulting allocation of resources would coincide with the de-

Permission to make digital or hard copies of all or part of this work for personal or classroom use is granted without fee provided that copies are not made or distributed for profit or commercial advantage and that copies bear this notice and the full citation on the first page. To copy otherwise, to republish, to post on servers or to redistribute to lists, requires prior specific permission and/or a fee. BICT 2017, March 15-16, Hoboken, United States

ISBN 978-1-63190-148-5

DOI: 10.4108/eai.22-3-2017.152405

Copyright $\odot 2017$ EAI
Examples of agents can be bacteria, insects as well as military ad hoc network consisting of mobile units consisting of several transceivers. In this scenario, resources are radio frequencies. This agents organized into groups that can be included in a higher order group, which may be included in a group of a higher order. It was necessary to develop an algorithm of the automatic deployment of such networks, taking into account the possible disconnections due to the movement of agents, as well as intentional or unintentional interference. This problem is closely related to the military communication network problem [7] whereby the organization of radio networks is planned between soldiers, squads, platoons, etc. Also similar problem arises in the organization of networks of UAVs, terrestrial mobile robots and other objects which have limited energy store. Consequently, it is necessary to split them onto clusters so that the amount of the data exchange in the each cluster would be much more than the amount of the data exchange between clusters.

Normally, it is necessary to set the frequency for the each agent in the network. The place of the agent in the hierarchy and its specific requirements should be taken into account. The number of agents and their devices may be a few thousand. Traditional methods of the radio network planning include construction of the detailed communication graph of networks and allocation of the frequencies by a human. Most modern algorithms include the allocation of frequencies through beacons exchange but all of them assume that agent have some knowledge about the whole system and that initial predefined frequencies for the beacons exchange exist.

In this paper we propose a different approach. Agents have the ability to classify each other by the signals that include the set of attributes which can be used for the mutual recognition of each other's role in the hierarchy. The more signals agent receives, the better it knows the hierarchy of agents, its own place in it and the global resources allocation. The purpose of each agent is to find the best frequency and attract agents of the same type while repelling other type agents. If we previously define the "requirements graph" (the ideal network) then we can score the quality of the resource allocation by the self-organization by measuring the difference between obtained and ideal network.

The idea of such a solution is inspired by mechanisms of mutual identification which have social insects and even humans. Especially the author was inspired by the behavior of hooded crows [2], which arranged their daily roll call outside his office. Every crow had a well-defined style of croaking and its place in the hierarchy. All crows strictly maintained a certain order. If the food had been detected then crows 
gathered together by croaking and tried to repel competing flock.

Earlier [6] the author examines the organization of the radio network on the a priori unknown communication channel by the mean of exchange of tuning beacons (TB) and response beacons (RB) between the centers of communication control (CCC) and radio stations which are tuned. The CCC sends TB to the selected channel of the preset channel grid, while the radio station scans this channel grid, and sends RB when TB is detected. The CCC sends the radio configuration data including the device frequency and other parameters after receiving the RB. Beacons exchange mechanism is established so that the communication network structure is built without a priori information about agents. This information is obtained on the basis of beacons which are transmitted by agents. Ultimately, the radio station found its place among the group of other stations without the human assistance.

Unlike the system presented in [6] which is eventually standard well-founded transition system, the system in the present paper is the so called "overlapped labeled transition systems" [10] because its agents can be simultaneously attracted by different resources. Moreover, agent can fall in the situation, where attracting and repelling stimuli have the equal strength. Finally, agent behavior can not be planned a priori because it depends on many unknown and unpredictable reasons.

\section{SYSTEM DESCRIPTION}

Give the formal description of the our resource allocation problem. Let

$$
A g=\left\{a g_{i} \mid i=\overline{1, n}\right\}
$$

is the set of agents,

$$
\mathcal{S} \subset \mathbb{R}^{m}
$$

is the set of the agent's features, $[0, T]$ is the closed interval of the agents' functioning time,

$$
\mathbf{I}[t]: A g \rightarrow \mathcal{S}
$$

is the injective map which assigns a feature vector to each agent in every $t \in[0, T]$. The feature's vector can include a position of an agent in the agent's hierarchy, characteristics of a resources found by an agent etc.

Define the "metric" in the set $\mathcal{S}$

$$
\rho: \mathcal{S}^{2} \rightarrow \mathbb{R},
$$

$\rho\left(\operatorname{sag}_{1}, \operatorname{sag}_{2}\right)=0$ iff $\operatorname{sag}_{1}=\operatorname{sag}_{2}, \rho\left(\operatorname{sag}_{1}, \operatorname{sag}_{2}\right) \geq 0, \operatorname{sag}_{1}$, $\operatorname{sag}_{2} \in \mathcal{S}$.

For example, agents correspond to wireless sensors or people, military units, moving together groups of small UAVs etc.

Define the signal sgn as a subset of the set $\mathcal{S}$.

Let

$$
\text { Chann }=\left\{\text { chann }_{i} \mid i=\overline{1, p}\right\}
$$

is set of resources (which are communication channels in the case of a telecommunication network) and Chann $[t] \subseteq$ Chann is the set of all resources available at the time $t \in$ $[0, T]$.

Define the function of an resource's attractiveness in the moment $t \in[0, T]$ :

$$
f_{\text {chann }}[t]: \text { Chann } \rightarrow \mathbb{R} .
$$

If resources are telecommunication channels then attractiveness corresponds to the data rate, signal to noise relation and similar characteristics.

Also define a function of the total capacity of a resource in the moment $t \in[0, T]$

$$
S_{c}[t]: \text { Chann } \rightarrow \mathbb{N} \text {. }
$$

This function determines how many agents can use the given resource simultaneously. Initially $S_{c}[t](\operatorname{chann})=0$ for all $t \in[0, T]$ and chann $\in$ Chann.

Each agent $a g \in A g$ can search for resources, capture resources, transmit signals and wait for signals. Also agents can accept three roles: an ordinary agent $C N$, an agent's flock leader $C C C$ and a flock of flocks leader $M C C C$. Suppose that agents with the role $M C C C$ search for resources in non-intersecting areas. Let

$$
f_{\text {role }}: A g \rightarrow \text { Role }=\{C N, C C C, M C C C\}
$$

is the injective map determining the agent' role.

Let $C_{1}, \varepsilon_{1}, \varepsilon_{2}[t] \leq S_{c}[t]$ are controllable parameters. Set the number of known agents using a resource chann in the moment $t N_{c}[t]$ (chann) $:=0$ for all $a g \in A g, t \in[0, T]$ and chann $\in$ Chann. The initial behavior of the agent's flock can be described as follows:

- agents $a g_{M}$ with $f_{\text {role }}\left(a g_{M}\right)=M C C C$ or $f_{\text {role }}\left(a g_{M}\right)=$ $C C C$ continuously search for the resources chann $\in$ Chann $[t]$ with $f_{\text {chann }}[t]($ chann $)>C_{1}$,

- if $a g_{M}$ locates resource in the moment $t_{0}$ and $N_{c}\left[t_{0}\right]<$ $\varepsilon_{2}\left[t_{0}\right]$ it sends the signal

$$
T B=\mathbf{I}\left[t_{0}\right]\left(a g_{M}\right) \in \mathcal{S},
$$

which consists of the information about the $a g_{M}$ and found resources,

- if agent $a g_{N}$ with roles $C N, C C C$ receives $T B$ in the moment $t_{1} \in[0, T]$ then if $\rho\left(\mathbf{I}\left[t_{1}\right]\left(a g_{N}\right), T B\right)<\varepsilon_{1}$ it attracts by the signal and transmits the signal $R B=$ $\mathbf{I}\left[t_{1}\right]\left(a g_{N}\right)$, else it repels by the signal and excludes resources in the TB from its search,

- if agent $a g_{M}$ with $f_{\text {role }}\left(a g_{M}\right)=M C C C$ or $f_{\text {role }}\left(a g_{M}\right)=$ $C C C$ detects in the moment $t_{2} \in[0, T]$ signal $R B$ from the agent $a g_{N}$ then if $N_{c}\left[t_{2}\right]<\varepsilon_{2}\left[t_{2}\right]$ then $a g_{M}$ sets $N_{c}\left[t_{2}\right]:=N_{c}\left[t_{2}\right]+1$ and sends to the $a g_{N}$ the signal $T D$ allowing the resource use.

We should notice that resource can accidentally disappear in the allocation process. For example, radio frequency can became too noisy or too overcrowded.

The difference between roles $C C C$ and $M C C C$ is that the agent with role $C C C$ can distribute individual resource but the agent with role $M C C C$ can distribute subsets of resources. Also atomic agents can form clusters and hierarchies on the base of the distance function $\rho$. The agents from one clusters should tend to share one resource. Such clusters can be viewed as agents of higher order.

Define a subagent as the part of agent which can use the specific type of resource. Denote the set of subagents as

$$
\mathrm{Comm}=\left\{\operatorname{comm}_{1}, \ldots, \operatorname{comm}_{q}\right\} .
$$

The example of a subagent is the specific transceiver assigned to a mobile unit. Define map $f_{\text {have }}[t]: C o m m \rightarrow$ 
$\mathrm{Ag}$ indicating the agent which own the subagent comm $\in$ Comm at the time $t \in[0, T]$.

The result of the resources allocation in the moment $t \in$ $[0, T]$ is the following entity:

Definition 1. The "communication graph" at the time $t \in$ $[0, T]$ is the labeled graph

$$
\Gamma_{\text {comm }}[t]=\left(V_{\text {comm }}[t], E_{\text {comm }}[t], \Lambda[t]\right),
$$

where

$$
V_{\text {comm }}[t]=C o m m \cup A g \cup \text { Chann }[t]
$$

are vertices, $E_{\text {comm }}[t] \subseteq\left(V_{\text {comm }}[t]\right)^{2}$ are nodes,

$$
\Lambda[t]: V_{\text {comm }}[t] \rightarrow \mathbb{R}
$$

is the node labeling function,

$$
\left.\Lambda[t]\right|_{\text {Chann }}=f_{\text {chan }}[t]: \text { Chann } \rightarrow \mathbb{R} .
$$

Define the "etalon variant" of the resource allocation as follows

Definition 2. The "requirements graph" is the labeled graph

$$
\Gamma_{r e q}=\left(V_{r e q}, E_{r e q}, \Lambda_{r e q}\right),
$$

$V_{r e q}=A g \cup R e q, E_{r e q}=V_{r e q}^{2}, R e q$ is the set of "resource templates" (abstract resources),

$$
\Lambda_{\text {req }}: V_{\text {req }} \rightarrow \mathbb{R}
$$

is the node labeling function (it corresponds to requirements for the quality of the channel between a pair of agents in the case of the telecommunication network).

The resource allocation procedure is successful if

$$
\mu\left(\Gamma_{\text {req }}, \Gamma_{\text {comm }}[t]\right) \rightarrow \min ,
$$

where $\mu$ is some graph distance, $t \in[0, T]$. We can assume that if $\varphi: \Gamma_{r e q} \rightarrow \Gamma_{\text {comm }}[t]$ is given and the restriction of the $\varphi$ on the subgraph $\Gamma_{r e q}^{\prime} \subset \Gamma_{r e q}$ is an isomorphism then

$$
\mu\left(\Gamma_{r e q}, \Gamma_{\text {comm }}[t]\right)=\frac{\left|\Gamma_{r e q} \backslash \Gamma_{r e q}^{\prime}\right|+\left|\Gamma_{\text {comm }}[t] \backslash \varphi\left(\Gamma_{r e q}^{\prime}\right)\right|}{\left|\Gamma_{r e q}^{\prime}\right|} .
$$

Examples of the $\Gamma_{r e c}$ and $\Gamma_{\text {comm }}$ is shown on the fig. 1.

Further we can replace all subgraphs like "chann $\in$ Chann and its immediate neighbors $e_{i}(\operatorname{chann}) "$ in the $\Gamma_{r e c}$ and $\Gamma_{\text {comm }}$ onto the full clique subgraphs of the vertices $e_{i}$ (chann) connected with edges marked by the comm and do not make difference between obtained and source graphs.

\section{DISTRIBUTION OF CHANNELS AND COM- MUNICATION DEVICES}

\subsection{Classical scheme of the channel assignment}

The classical and wide-using scheme of the channel assignment is quite opposite to the algorithm described above. At the first stage we have the "requirements graph" of the communication system $\Gamma_{r e q}$, at the second stage we construct the "communication graph" $\Gamma_{c o m m}$ from $\Gamma_{r e q}$ with help of various rules and at the third stage we calculate the set of radio frequencies from the $\Gamma_{\text {comm }}$ and input frequencies from this set into communication devices (if there are no functioning telecommunication channels $\Gamma_{\text {comm }}[t]$ actually is sent on the flash drive). Briefly describe the rules of the constructing $\Gamma_{\text {comm }}$.

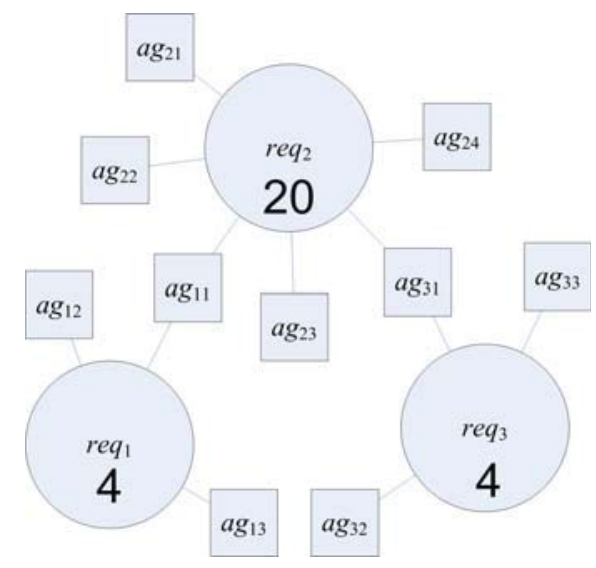

(a)

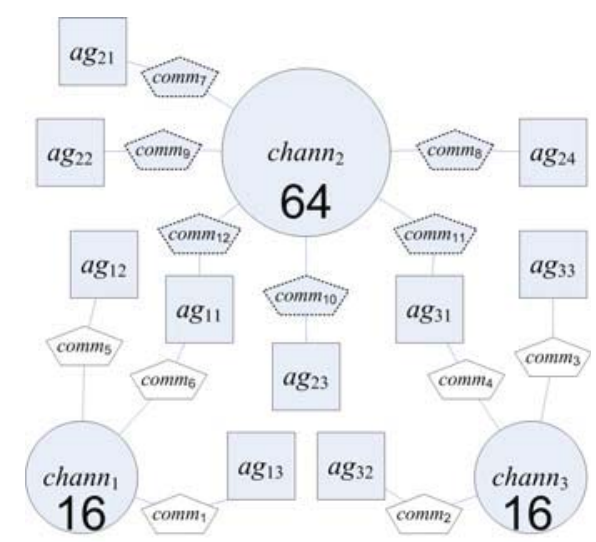

(b)

Figure 1: The example of the $\Gamma_{r e c}(\mathrm{a})$ and the corresponding $\Gamma_{\text {comm }}$ (b) graphs

- One vertex in the "requirements graph" corresponds to at least one vertex in the "communication graph" of the same type (agents correspond to agents, resources templates correspond to resources).

- One edge $e_{r} \in E_{r e q}$ in the "requirements graph" corresponds to the edge $e_{c} \in E_{\text {comm }}[t]$ or to the continuous path in the "communication graph".

- If the vertex $r e q \in R e q$ of the $\Gamma_{r e q}$ corresponds to the set of vertices Chann $J=\left\{\right.$ chann $_{j} \in$ Chann $\mid j \in J \subset$ $\mathbb{N}\}$ of the $\Gamma_{\text {comm }}$ then

$$
\frac{\min _{j \in J} f_{\text {chann }}[t]\left(\text { chann }_{j}\right)}{N_{c}[t]} \geq \Lambda_{r e q}(\text { req }),
$$

where $N_{c}[t]$ is the number of communication devices using the channel chann.

This scheme is quite common but has a number of disadvantages:

- The "requirements graph" is rigidly tied to a particular set of agents. If it is necessary to go to another set of agents with a similar structure, for example, from 1st infantry regiment of the 1st infantry division to 2nd infantry regiment of the 1st infantry division, the whole process of constructing graphs $\Gamma_{r e q}$ 
and $\Gamma_{\text {comm }}[t]$ should be repeated, which can be quite difficult, both in terms of human and computing time costs.

- The "communication graph" is rigidly associated with particular devices but not with types of devices that requires the complete rebuilding of the graph in the case of replacing of some communication devices.

- Algorithm is tied onto the planning center. The center is required to enter "requirements graph" and perform construction of the "communication graph". All other network nodes inoperative before the end of the planning and delivery of the communication graph, and this situation repeats each time when it is necessary to change the "communication graph".

A changes in graphs $\Gamma_{r e q}$ and $\Gamma_{\text {comm }}[t]$ can occur during operation of the system of agents. In practical applications, usually changes in the $\Gamma_{r e q}$ occur slowly and entail changes in the $\Gamma_{\text {comm }}[t]$. The graph $\Gamma_{\text {comm }}[t]$ may change very frequently at the constant graph $\Gamma_{r e q}$. Changes of the $\Gamma_{\text {comm }}[t]$ depend on many factors: the state of communication devices, speed of agents, the landscape on which agents move, availability of communication channels.

\subsection{Further development of the role-oriented scheme of the channel assignment}

\subsubsection{The general idea}

We can use the constant graph $\Gamma_{r e q}$ instead unpredictable graph $\Gamma_{\text {comm }}[t]$ and make different realizations of the graph $\Gamma_{\text {comm }}[t]$ automatically. Therefore the $\Gamma_{\text {req }}$ can be viewed as the generator of the infinite stream of graphs $\Gamma_{\text {comm }}[t]$. The question naturally arises from the preceding paragraph: is it possible to define the requirements graph $\Gamma_{r e q}$ not as a priori information (which may be difficult for networks of hundreds and thousands of complexly organized agents) and to build it automatically? This is an intermediate stage between the fully automatic resource allocation mentioned at the beginning of the paper and the standard way of the channel assignment.

Obviously, the answer to this question is "yes" if we provide the formalized set of rules that allows to build $\Gamma_{r e q}$ from the

$$
\left(\rho, \text { Ag, Role, } f_{\text {role }}\right) .
$$

Definition 3. We define requirements generator as expression like

$$
\begin{gathered}
G\left[f_{\text {role }}, \rho\right]\left(a g_{k_{1}}, \ldots, a g_{k_{l}}, \text { role }_{k_{1}}, \ldots, \text { role }_{k_{l}}\right) \rightarrow \\
\exists(r \in \operatorname{Req}) \forall(i=\overline{1, l}) \quad\left(a g_{k_{i}}, r\right) \in E_{r e q} \& \Lambda_{r e q}(r)=\alpha,
\end{gathered}
$$

where

$$
G\left[f_{\text {role }}, \rho\right]: A g^{n} \times \text { Role }^{n} \rightarrow\{0,1\}
$$

are $2 n$-ary predicate. The expression in the left part of the requirements generator $G$ we will call the "input statement" and the expression in the right part of $G$ - the "output statement" about the agents.

In fact we can express the classical channel allocation problem and the problem described in the section 2 in the terms of fuzzy logic. We have the fuzzy set of graphs $\Gamma_{r e q}$ and find the graphs $\Gamma_{\text {comm }}$ which belongs to the $\Gamma_{r e q}$ in the first case. In the second case we actually construct the fuzzy set of graphs $\Gamma_{r e q}$ in co-inductive manner on the basis of various fragments of $\Gamma_{\text {comm }}$ already known and after that search for the complete $\Gamma_{\text {comm }}$ belonging to the $\Gamma_{\text {req }}$. The similar co-inductive construction was developed in the [9] for designing an unconventional computer based on the medium of slime mould.

\subsubsection{Rule definition language}

Denote that metric (1) can be defined through a tree of agents hierarchy $\Psi$ where the highest in the hierarchy agent is the root. Therefore certain properties of agents can be formalized in terms of $\Psi$ properties. A tuple ( $\Psi, A g$, Role, $\left.f_{\text {role }}\right)$ is obviously generates a subgraph of the $\Gamma_{\text {req. }}$.

It is possible to define relationships " $\geq$ " and " $\leq$ " between agents in the tree $\Psi$. We say that $g_{1} \leq g_{2}$ for agents $a g_{1}$, $a g_{2} \in A g$ if the shortest path from $g_{2}$ to the root element of the tree $\Psi$ is shorter than the shortest path from $g_{1}$ to the root element of the tree $\Psi$ or lengths of this paths are equal. If the shortest path from $g_{2}$ to root element of the tree $\Psi$ is longer than the shortest path from $g_{1}$ to the root element of the tree $\Psi$ or lengths of these paths are equal then we will state that $g_{1} \geq g_{2}$. Further, in the tree $\Psi$ it is possible to specify join operation " $\vee$ " such as $g_{1} \vee g_{2}=$ $\min \left\{g \in A g \mid g_{1} \leq g \& g_{2} \leq g\right\}$ (i.e., as the lowest common ancestor of $g_{1}$ and $g_{2}$ ), thus determining the bounded upper semilattice $(A g, \vee)$

Assume that the center of the communication system planning has the rule definition language interpreter and user can define a set of rules in the form of the set of requirements generators, save this set to a file or load this set from a file. The user will obtain the graph $\Gamma_{r e q}$ in the form of the set of output statements about agents by calling requirements generators $G r e q \_i$ from the set of generators.

\subsubsection{The transition from the agent to agent descrip- tors}

The resource assignment in our paper is still tied to the agents. It is inconvenient if we need to go from a structure containing some agents to the same structure which should contain other agents. Way out is using of agents classes instead agents.

Definition 4. Let $\mathcal{P}(x)=\left\{P_{i}(x) \mid i=1, \ldots, q\right\}$ is set of input statements about the agent $x \in A g$. If

$$
\forall(i=1, \ldots, q) P_{i}\left(a g_{1}\right)=P_{i}\left(a g_{2}\right)
$$

then say that agents $a g_{1}, a g_{2} \in A g$ are equivalent and write $a g_{1} \stackrel{\mathcal{P}}{\sim} a g_{2}$

The relation $\stackrel{\mathcal{P}}{\sim}$ will also be denoted as a $\sim$. All equivalent agents can be considered exactly the same in the process of construction of the requirements graph $\Gamma_{r e q}$, so it is possible to part the set $A g$ into equivalence classes. Hence we move from (3) to the map

$$
\tilde{G}\left[f_{\text {role }}, \rho\right]:(A g / \sim)^{n} \times \text { Role }^{n} \rightarrow\{0,1\} .
$$

Definition 5. We say that a pair desc $=(x, i), x \in(G r / \sim$ ), where $i$ is the ordinal number of the agent $a g_{i}$ from $x$ is the descriptor of the agent $a g_{i}$. Denote the set of descriptors by $\mathcal{D}$. 
It is obvious that the descriptor of agent $a g_{n}$ from the class $x \in A g / \sim$ is uniquely determined and as $(\mathcal{P}(x), n)$.

Summing up, we replace the $\Gamma_{r e q}$ by the object $\tilde{\Gamma}_{r e q}$, in which agents are replaced by respective classes of agents. The channel distribution algorithm for such entities is exactly the same as described in the preceding paragraphs, but without reference to specific numbers (ids) of agents. Therefore, $\tilde{\Gamma}_{r e q}$ generates graphs $\Gamma_{r e q}$ as $\Gamma_{r e q}$ generates $\Gamma_{\text {comm }}$.

Note that the algorithm is still tied to the specific communication devices. It is also possible to get away from this. Let the set of communication devices is part into disjoint classes of communication devices, family of such classes we denote as $\mathcal{C}=\left\{\right.$ Comm $\left._{i}\right\}, \cup_{i}$ Comm $_{i}=$ Comm. If comm $_{1}$, $\operatorname{comm}_{2} \in \mathrm{Comm}_{i}$ and $\operatorname{comm}_{1}, \mathrm{comm}_{2}$ belong to the same agent (it is written as $f_{\text {have }}\left(\mathrm{comm}_{1}\right)=f_{\text {have }}\left(\mathrm{comm}_{2}\right)$ in this formalism) then assume that communication devices $\mathrm{comm}_{1}, \mathrm{comm}_{2}$ are equivalent and write $\mathrm{comm}_{1} \sim \mathrm{comm}_{2}$, . The factor-set Comm/ can be used instead of the Comm in the construction of the communication graph, i.e., we can use graph $\tilde{\Gamma}_{\text {comm }}[t]$ instead of $\Gamma_{\text {comm }}[t]$.

Just as we have built graphs $\tilde{\Gamma}_{r e q}$ and $\tilde{\Gamma}_{\text {comm }}$ from graphs $\Gamma_{\text {req }}$ and $\Gamma_{\text {comm }}$ reverse operation may be done. If we substitute classes from $\mathrm{Gr} / \sim, \mathrm{Comm} / \sim$ by arbitrary representatives of these classes, then graphs $\tilde{\Gamma}_{r e q}$ and $\tilde{\Gamma}_{\text {comm }}$ results in graphs $\Gamma_{r e q}$ and $\Gamma_{\text {comm }}$. At the same time it is necessary to ensure that if any class of $\mathrm{Ag} / \sim, \mathrm{Comm} / \sim$ is present in the corresponding graph more than once, then pairwise distinct representatives should be chosen for the each replacement case.

Summing up, the relationship expressed by the diagram in fig. 2 exists among all the aforesaid graphs:

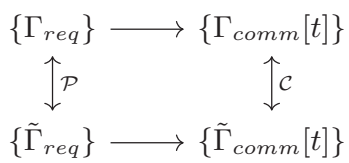

Figure 2: The relationship between the requirements graph and the communication graph

\subsubsection{The simple example}

Illustrate aforesaid with example. Let the agents on the fig. 1 have the following features vectors:

$$
\begin{gathered}
\mathbf{I}[t]\left(a g_{i j}\right)=(i, j, 0,1,0), i=1,3, j=2,3 \\
\mathbf{I}[t]\left(a g_{2 j}\right)=(2, j, 0,0,1), j=\overline{2,4} \\
\mathbf{I}[t]\left(a g_{i 1}\right)=(i, 1,1,1,1), i=\overline{1,3}
\end{gathered}
$$

The 1st component of the features vector can be, for example, a number of a platoon, the 2nd - a number of an agent inside its platoon, the 3rd is the sign of leadership, the 4 th is the sign of the ownership of the device type 1 , the 5 th is the sign of the ownership of the device type 2 . Define distance between the agents with features vectors $s a g_{1}$, $\operatorname{sag}_{2} \in \mathcal{S} \subset \mathbb{R}^{5}$ as

$$
\begin{gathered}
\rho\left(\operatorname{sag}_{1}, \operatorname{sag}_{2}\right)=\frac{14}{4}\left|\operatorname{sag}_{1}^{1}-\operatorname{sag}_{2}^{1}\right|+\frac{1}{4}\left|\operatorname{sag}_{1}^{2}-\operatorname{sag}_{2}^{2}\right|+ \\
+\left|\operatorname{sag}_{1}^{3}-\operatorname{sag}_{2}^{3}\right|+\left|\operatorname{sag}_{1}^{4}-\operatorname{sag}_{2}^{4}\right|+\left|\operatorname{sag}_{1}^{5}-\operatorname{sag}_{2}^{5}\right|+ \\
+\theta\left(1-\operatorname{sag}_{1}^{3} \operatorname{sag}_{2}^{3}\right) \theta\left(\left|\operatorname{sag}_{1}^{1}-\operatorname{sag}_{2}^{2}\right|\right)
\end{gathered}
$$

where $\theta(x)=1, x>0, \theta(x)=0, x<0$. If $\rho\left(\operatorname{sag}_{1}, \operatorname{sag}_{2}\right) \leq$ $3 \frac{3}{4}$ then assume that agents $a g_{1}, a g_{2}$ try to go to the same resource (i.e. form cluster). Such rule easily gives $\Gamma_{\text {req }}$ on the fig. 1a. If we state that agents $a g, \mathbf{I}(a g)[t]=$ $\left(s a g^{1}, s a g^{2}, s a g^{3}, s a g^{4}, s a g^{5}\right)$ with $s a g^{5}=0$ can use resources labeled by " 20 " on the $\Gamma_{r e q}$ only and agents with $s a g^{4}=0$ can use resources labeled by "4" only then we obtain $\Gamma_{\text {comm }}$ on the fig. $1 \mathrm{~b}$.

We can see that the aforesaid problem look similar to the clustering problem[3] which is known in the machine learning.

\section{AGENT'S ROLE-ORIENTED SELF - ORGANIZING TELECOMMUNICATION SYSTEM}

This section will describe the communication system that supports fully automatic decentralized generation of networks and channel assignment. Communication devices in this system form the three-level hierarchy. There also will be given some time characteristics of the decentralized channel assignment algorithm.

\subsection{Minimal requirements}

Assume that channels which we distribute are frequency channels and communication devices have two transceivers connected by a common router. One of the transceivers (device $A$ ) is required to scan channels from the predefined technological grid of radio frequencies and to receive beacons, the second (device B) is required to send beacons. In subsequent phases of the proposed algorithm one of the transceivers will interact with higher centers, and other with subordinate stations. The idea of such a device in more detail is described in $[1]$.

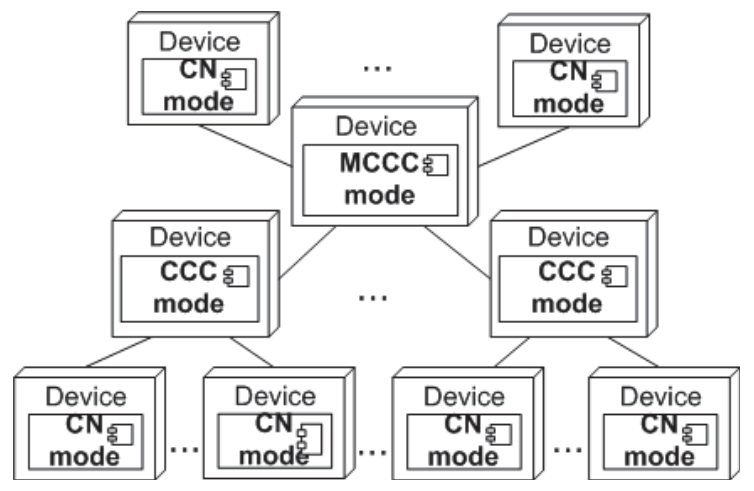

Figure 3: Scheme of the hierarchical self-organizing network

\subsection{Algorithm}

Each communication device have the same technological radio frequency grid Chann consisting of $m$ frequency chan- 
nels. Each device can act in three roles: as the main communication control center (MCCC), the communication control center ( $\mathrm{CCC})$, the communication node $(\mathrm{CN})$. In each role, communication devices operate differently (corresponding UML state machine diagram is shown in fig. 4).

Each device with number $i$ have device descriptor desc $_{i}=$ $(x, i) \in \mathcal{D}$ and the predicate of the descriptor testing $P_{i}$ : $G r / \sim \rightarrow\{0,1\}$. The predicate is necessary to check whether the device with some descriptor belongs to the same group of devices with device $i$. Also, devices with roles MCCC and CCC must contain, at minimum, the tree $\Psi$ and the generator of requirements $G$.

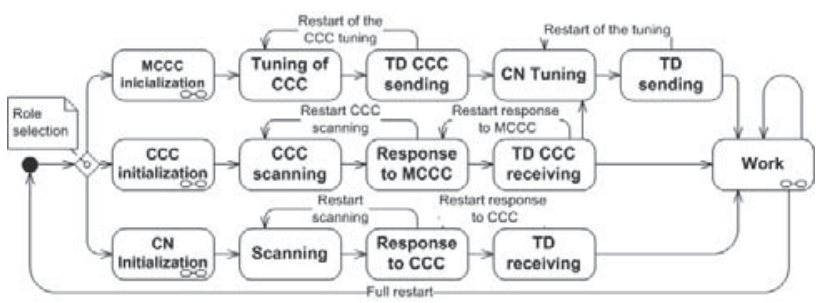

Figure 4: Statechart of the communication device

Devices exchange messages in the process of configuration. This messages include for the sender with number $i$ :

- tuning beacon of the MCCC (TB MCCC), which includes, at a minimum, the beacon type code, number of the beacon and response channel $f_{r x}^{0}$,

- tuning beacon (TB), which includes the beacon type code, the serial number of tuning beacon $N_{i}$, the descriptor of the sender $\operatorname{desc}_{i}$, the response channel number $f_{r x}^{0}(i)$,

- response beacon of the $\mathrm{CCC}$ ( $\mathrm{RB} \mathrm{CCC}$ ) and response beacon (RB), which include the beacon type code, the serial number of the response beacon $N_{i}$, the descriptor of the sender $\operatorname{desc}_{i}$,

- packets of the configuration data for the CCC (TD CCC) and packets of the configuration data (TD), which may include the sender's address, the destination address, separate frequencies or frequency ranges intended for the recipient.

Device with number $j$ in MCCC mode:

1. Initializes itself:

(a) Sequentially scans frequencies from Chann and ranks Chann by noise level or by SNR.

(b) Selects the best transmit channel $f_{t x}^{0} \in$ Chann and the best receive channel $f_{r x}^{0} \in$ Chann.

(c) Tunes device A to channel $f_{t x}$, device B to channel $f_{r x}$.

2. Tunes all CCC. MCCC transmits TB CCC, which includes $f_{r x}^{0}$, on the channel $f_{t x}^{0}$. This stage lasts until receiving at $f_{r x}^{0} \mathrm{RB}$ CCC from the predefined number $p$ of tuned CCCs.
3. Sends configuration data to $\mathrm{CCC}$. After receipt of $\mathrm{RB}$ CCC from $p$ CCC, MCCC divides the set of available channels Chann to fragments Chann $_{i}, i=1, \ldots, p+1$ and sends information about each fragment $C$ hann $n_{i}$ to $i$ th CCC. MCCC reserves the fragment Chann $_{p+1}$ for itself. In addition, MCCC may send to CCC fragments of the graph $\Gamma_{r e q}$. If after a specified timeout specified number of RB CCC has not been received, MCCC returns to the state "setting CCC".

4. Tunes directly subordinate to the MCCC devices. For this purpose MCCC configures device A to the best frequency $f_{t x}^{1} \in$ Chann $_{p+1}$, devices B to the next best frequency $f_{r x}^{1} \in$ Chann $_{p+1}$ and transmits TB containing information about $f_{r x}^{1}$ and MCCC descriptor. This process repeats until receiving on $f_{r x}^{1} k \mathrm{RB}$ such that for desc $_{i}$, received from $i$-th beacon, $P_{j}\left(\right.$ desc $\left._{i}\right)=1$. If after a specified timeout specified number of $\mathrm{RB}$ is not received, then MCCC returns to the state "tuning device".

5. Switches to the "work" state in which it transmits user data, maintains network connectivity, finds routes, builds clusters etc.

In $\mathrm{CCC}$ mode communication device with number $j$ :

1. Initializes itself:

(a) Sequentially scans frequencies from Chann and ranks Chann by a noise or SNR.

(b) Selects the best channel $f_{0} \in$ Chann to start scanning the channel grid.

2. Scans Chann, i.e. sequentially tunes device A to the channels from the Chann, ordered by the noise level or by the $S N R$, starting from $f_{0}$, and stops at each channel for the time which is required for receiving TB MCCC. The process of scanning stops when TB $\mathrm{MCCC}$ is received.

3. Responds to the MCCC, for which determines the start time of the tuning cycle $t_{0}$ from the number $N_{i}$ of the TB and transmits RB CCC to the channel $f_{r x}$, obtained from TB CCC at the time $t=T_{0}+j T_{1}$, where $T_{1}$ is the period of time shared by all devices.

4. Receives from MCCC the configuration data, including a subset of channels Chann . $_{\text {. }}$.

5. Tunes directly subordinate devices (similar to MCCC) i.e. transmits TB on the best rate channel Chann $n_{i}$. If the $\mathrm{CCC}$ receives from communication nodes the predetermined number of RBs, such that for the desc $c_{i}$, derived from the $i$-th response beacon, it follows that $P_{j}\left(\right.$ desc $\left._{i}\right)=1$ then CCC completes setup process.

6. Sends configuration data to directly subordinate communication nodes, similar to MCCC.

7. Switches to the "work" state, similar to MCCC.

In $\mathrm{CN}$ mode communication device with number $j$ :

1. Initializes itself similar to CCC.

2. Scans the Chann set, similar to the CCC. Scanning is completed upon detection of TB from its CCC. 
3. Sends RB to its CCC and expects configuration data.

4. Receives the configuration data from its CCC (i.e. from one that its descriptor $\operatorname{desc}_{i}$ corresponds to the rules specified in this node, $\left.P_{j}\left(\operatorname{desc}_{i}\right)=1\right)$.

5. Switches to the "work" state.

We see that in the process of beacon exchange of devices with roles MCCC and CCC descriptors of certain groups of other devices become known. According to the obtained descriptors and to contained in the device graph $\tilde{\Psi}$ and generator of requirements $G$, each CCC constructs graph $\tilde{\Gamma}_{r e q}^{j}$ by the algorithm described in the section3.2.3, $\cup_{j} \tilde{\Gamma}_{\text {req }}^{j}=$ $\tilde{\Gamma}_{\text {comm }}$. Further, CCC constructs graph $\Gamma_{\text {comm }}^{j}$ by the selected for this CCC fragment Chann $n_{j}$ of the set of channels and by the graph $\tilde{\Gamma}_{\text {req }}^{j}$. Due to the fact that fragments of the set of channels do not overlap, $\Gamma_{\text {comm }}=\cup_{j} \Gamma_{\text {comm }}^{j}$ satisfies the requirements set out in the section 3.1. Graphs $\Gamma_{\text {comm }}^{j}$ are TD packets which will be sent to $\mathrm{CN}$.

Thus, the mutual identification of agents and descriptor sharing takes place through the exchange of $\mathrm{TB}, \mathrm{RB}, \mathrm{TB}$ CCC, RB CCC (Fig. 5). Also this mechanism may be supplemented by authentication, described in [6]. In this method of authentication TB CCC and TB contains a public key and the RB $\mathrm{CCC}, \mathrm{RB}$ contain a session key which is encrypted with the public key, i.e., it is possible to use hybrid cryptosystem.

We can use the mechanism of frequency adaptation in the case of interference in the exchange of beacons process. For example, in the absence of RB, RB CCC for a long time, the process of configuring the $\mathrm{CCC}$ or node should be restarted, and the old channel $f_{t x}$ is excluded from the Comm for a certain time. If MCCC or one of the CCC becomes inaccessible to nodes, nodes can take the decision to change the role to MCCC or CCC.

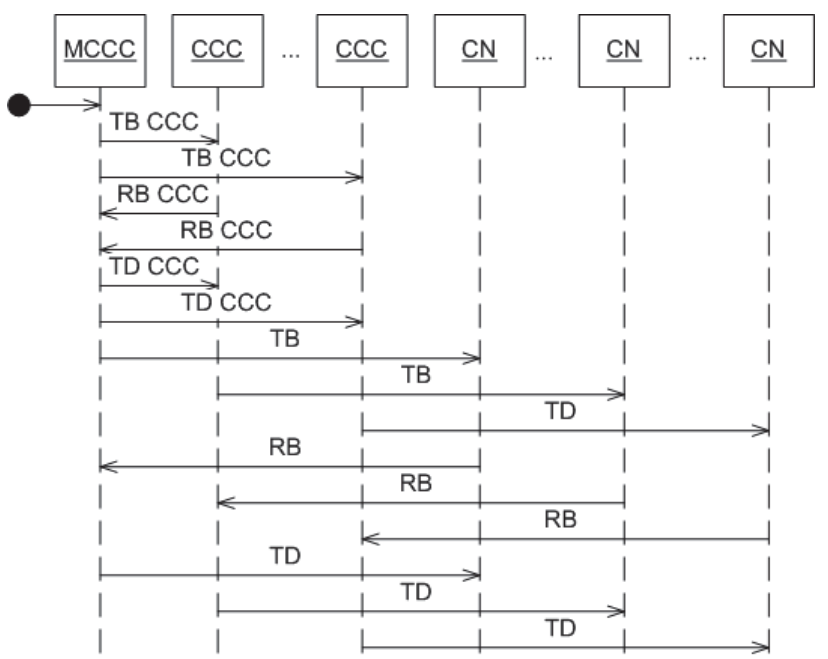

Figure 5: Sequence diagram of the beacons exchange

\subsection{Simulation of automatic tuning algorithm}

We modeled the aforesaid network in the OMNeT ++5.0 discrete event simulator. Dependence of network configuration completion time from the number of devices, number of frequency channels and the data rate was simulated. It was assumed that the network has one MCCC and several CCC, each of which had an equal number of subordinated devices. It was assumed that the length of all beacons is 24 bytes, the tuning beacon expected in the scanning process during $t_{w}=3 \cdot 8 \cdot 24 / v$ seconds, where $v$ is bit rate, bit error probability less than $10^{-3}$, communication devices start time is a uniformly distributed random value in the range $\left[0, N \cdot t_{w} / 2\right]$, where $N$ is a number of channels. Dependences depicted in the graphics 6,7 were obtained. The curves are shown in the $6(\mathrm{a}), 6(\mathrm{~b})$ was calculated on the assumption that one cluster contains 20 devices and three clusters are presented.

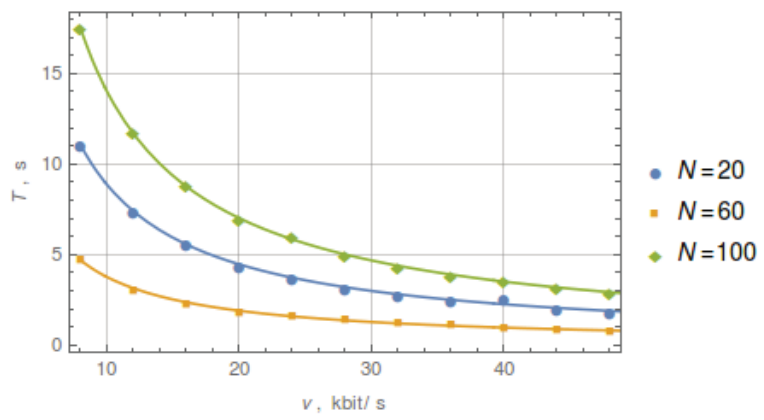

(a)

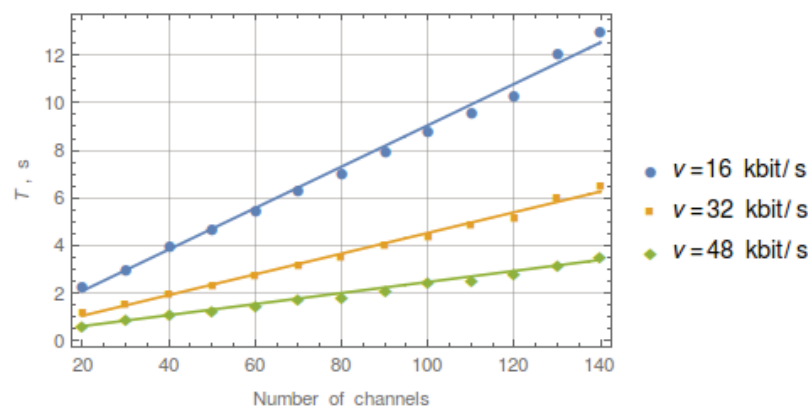

(b)

Figure 6: Dependence between the end of the network configuration time $T$, the data rate $v$ and the number of channels $N$

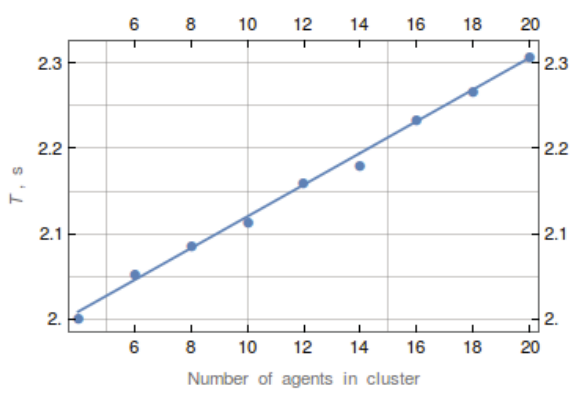

Figure 7: Dependence between the end of the network configuration time $T$ and the number of agents in the cluster, $v=32 \mathrm{kbit} / \mathrm{s}, N=60$

\section{CONCLUSION}


We developed the general mathematical formalism to describe the networks organized with a focus on the hierarchy of the network nodes and showed the use of such formalism for the specific self-organizing ad hoc network. The formalism includes the generation of graphs streams and allocation of resources without a priori information. In fact, said mathematical tool can be applied not only to the automatic organizing of telecommunication communications networks, but also to computer modeling of the ordinary non-automatic channel assignment as well as to many other social systems.

We compared three cases: the full self-organization without a priori information about resources, the partial selforganization and the "manual" resource allocation.

It is planned to produce a simulation of the dependence of the network's configuration completion time from movement speed of network nodes and from landscape properties using the motion model, referred to in article [4]. Further it is planned to use the proposed algorithms for modeling the behavior of the communications network in combat action using the model [5]. In this case, we can represent the resource allocation process and conflict at whole as a payoff cellular automaton based reflexive game introduced in [8].

\section{REFERENCES}

[1] V. Bessonov and A. Kuznetsov. The technology for creating ad hoc radio network with functions of cognitive radio on the principles of software defined radio. In CET 2014 XV International Scientific and Technical Conference "Cybernetics and High Technology of the XXI Century", pages 176-187. Voronezh: SPC "Sakvoee" LLC, May 2014. [in Russian].

[2] V. Grabovsky. The social structure of a population of hooded crows (corvus cornix) on the lenin hills in moscow. Zoologicheskii zhurnal, 3(62), 1983. [in Russian].

[3] A. Jain, M. Murty, and P. Flynn. Data clustering: A review. ACM Computing Surveys, 31(3):264-323, 1999.

[4] A. Kuznetsov. A model of the joint motion of agents with a three-level hierarchy based on a cellular automaton. Computational mathematics and mathematical physics, 57(2):339-349, 2017.

[5] A. Kuznetsov. The simplified model of military engagement based on the cellular automaton. Journal of Computer and Systems Sciences International, 56(3), 2017. To appear.

[6] A. Kuznetsov and S. Zharkov. A special wireless network configuration over a secure radiochannnel. Electrosvyaz, (12):28-35, 2016. [in Russian].

[7] J. Redi and R. Ramanathan. The darpa wnan network architecture. In 2011 - MILCOM 2011 Military Communications Conference, pages 2258-2263, Nov 2011.

[8] A. Schumann. Payoff cellular automata and reflexive games. J. Cellular Automata, 9(4):287-313, 2014.

[9] A. Schumann. p-adic valued logical calculi in simulations of the slime mould behaviour. Journal of Applied Non-Classical Logics, 25(2):125-139, 2015.

[10] A. Schumann. Towards context-based concurrent formal theories. Parallel Processing Letters, 25(01):1540008, 2015. 